\title{
Error Concealment for INTRA-frame Losses over Packet Loss Channels
}

\author{
Mengyao $\mathrm{Ma}^{\dagger}$, Oscar C. $\mathrm{Au}^{\ddagger}$, Liwei Guo ${ }^{\ddagger}$, \\ Zhiqin Liang ${ }^{\ddagger}$, and S.-H. Gary Chan ${ }^{\dagger}$ \\ $\dagger$ Dept. of Computer Science and Engineering \\ $¥$ Dept. of Electronic and Computer Engineering \\ Hong Kong University of Science and Technology \\ \{myma, eeau, eeglw, zhiqin, gchan\}@ust.hk
}

\begin{abstract}
In this paper, we propose an Error Concealment algorithm for INTRA-frame losses over packet loss channels. The novelty is that not only the INTRA-frame but also the subsequent INTER-frames are error concealed. We use the received INTRA-MBs to refine their neighbors based on the strong correlation between adjacent pixel values. In addition, Motion Compensation is used to reconstruct the INTER-pixel which has an INTRA-pixel in its motion trajectory. Both subjective and objective simulation results are given to demonstrate the performance of our proposed algorithm.
\end{abstract}

Key words: Error Concealment, Error Propagation, Motion Compensation, Spatial Interpolation

\section{Introduction}

Delivering video of good quality over the Internet or wireless networks is very challenging today, due to the use of predictive coding and Variable Length Coding (VLC) in video compression [1]. In block-based video coding method, if we use INTER-prediction mode, each macroblock (MB) is predicted from a previously decoded frame by Motion Compensation. If data loss occurs during the transmission, the corresponding frame will be corrupted, and this error will propagate to the subsequent frames because of INTER-prediction, until the next INTRAcoded frame is correctly received. In addition, a simple bit error in VLC can cause desynchronization; as a result, all the following bits cannot be used until a synchronization code arrives. Due to these facts, it is useful to develop some Error Resilience (ER) or Error Concealment (EC) techniques to control the errors in video transmission. Error resilience is usually applied at the encoder side. The coding efficiency of an ER codec is lower than a normal codec, because the encoder needs to introduce some redundancy to the stream. In the case of error, the decoder would use this additional information to reconstruct the video. On the other hand, error concealment is applied at the decoder side. It requires no change to the encoder and does not increase the bit rate, so it is more preferable for low bit-rate applications [2][3]. 
Lots of EC algorithms have been developed for video communication, such as spatial interpolation using some smoothness measure and temporal compensation based on inter-frame correlation [4][5]. Boundary matching algorithm (BMA) is also developed to estimate the lost motion vectors (MV) [6][7]. Most of current EC methods assume that only a few MBs or slices in a video frame are lost. However, in low bit-rate applications, one frame is usually carried in one data packet in order to save transmission overhead. As a result, the loss of one packet will lead to the loss of one entire frame [3]. When frame loss occurs, we can copy the previous received frame to reconstruct the lose one. More sophisticated methods recover the motion vectors (MVs) in pixel or block level based on the assumption of translational motion, i.e. motion remains constant along motion trajectory [3][8][9].

As in most of the block-based video coding systems all the INTER-frames are encoded based on the preceding INTRA-frame, the protection and restoration of INTRA-frames is especially important for the decoding of subsequent frames. However, as far as we know, most of the EC algorithms in the literature focus on the restoration of INTER-frames, and only a few works deal with the EC of INTRA-frames. In addition, almost all these algorithms assume that only part of the INTRA-frame is corrupted so that the lost MBs can be reconstructed using the information from the neighbors [10][11]. Since in low bit-rate video transmissions the loss of a packet usually results in the loss of a whole frame, an error concealment algorithm for INTRA-frame losses is necessary in reality. In this paper, we will focus on this problem and propose an algorithm to improve the reconstructed video quality when INTRA-frame loss occurs. The novelty is that not only the INTRA-frame but also the subsequent INTER-frames are refined using the received INTRA-MBs.

Random INTRA Refresh (RIR) scheme has been used in both earlier and current standards such as MPEG-4, H.263 and H.264, where INTRA-coded MBs are randomly inserted into the bitstream to remove artifacts caused by error and INTER-prediction drift. Although coding efficiency is reduced a little, RIR with a low INTRA-rate is more practical than inserting periodic INTRA-frames due to the bit-rate constraint [12]. As the RIR scheme is implemented in the encoder and does not introduce any decoding overhead, it is often jointly used with other ER or EC schemes. In our algorithm we assume that the received bitstream contains such INTRA-MBs. When an INTRA-frame is lost, the received INTRA-MBs in the subsequent frames can be used to refine their INTER-neighbors based on the strong correlation between adjacent pixel values. In addition, an INTER-pixel can also be refined by Motion Compensation (MC) if there is an INTRA-pixel in its motion trajectory.

The rest of this paper is organized as follows. In Section 2, we describe the proposed EC algorithm. Its performance is demonstrated in Section 3 by both subjective and objective results. Section 4 is conclusion. 


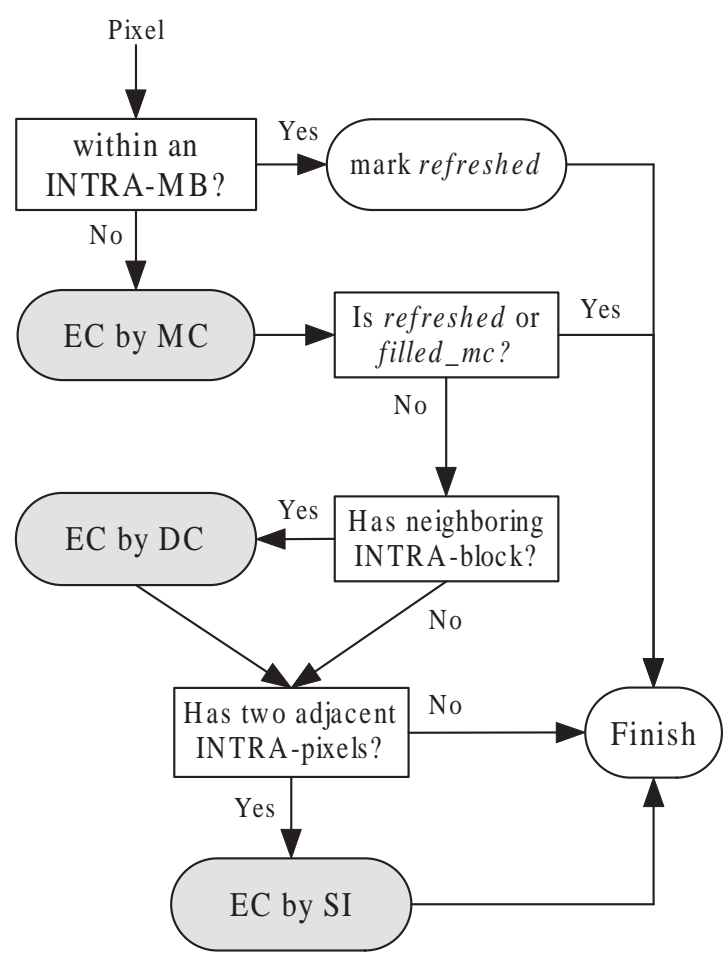

Fig. 1. The flow chart of the proposed EC algorithm for INTRA-frame loss.

\section{The proposed EC algorithm}

In conventional EC algorithms, only the corrupted (lost) frames are error-concealed. Although the subsequent frames can be decoded as usual, some annoying artifacts will exist due to the drifting errors and the video quality can be even worse in the case of INTRA-frame loss. In this work, we propose to use four ways to reconstruct the subsequent INTER-pixels after a lost INTRA-frame:

- Decoding directly as in the conventional codec;

- Error concealment by motion compensation (MC);

- Error concealment by the DC of INTRA-MB (DC);

- Error concealment by spatial interpolation (SI).

Each INTER-frame is decoded and then error concealed pixel by pixel, using the algorithm in Figure 1. We will describe the three EC ways in the following subsections and then summarize the algorithm. As the INTRA-MBs coded by Random INTRA Refresh (RIR) can help to stop the propagated error, for each pixel we use one mark to represent whether it is error-free (refreshed) or not. For a lost frame, all the pixels are set to be non_refreshed. And when an INTRA-MB is received later, the corresponding pixels are marked refreshed. So a status map 
$\left(M_{f}\right)$ needs to be maintained for each frame in the frame buffers, one bit for one pixel. In addition, we also maintain a small map $M_{s}($ size $16 \times 16)$ for the pixels in an INTER-MB. The initial status of each pixel is non_filled_mc. Whenever a pixel is refined by $\mathrm{MC}$, its status in $M_{s}$ is changed to filled_mc.

\section{$2.1 \quad$ EC by $\mathrm{MC}$}

Suppose there are $L$ frames in the reference frame buffer. For each INTER-pixel $p$, we have its motion vector $M V_{0}$ and the corresponding reference frame index $k_{0}, k_{0} \in\{1,2, \ldots, L\}$. Then $p$ can be refined by motion compensation (MC) if there is a refreshed pixel in its motion trajectory. In detail,

1. Mark the status of $p$ in $M_{s}$ as non_filled_mc. Use $M V_{0}$ to find the reference pixel of $p$, i.e. $q_{0}$. If $q_{0}$ lies at an integer-pixel position marked as refreshed, or if $q_{0}$ lies at a sub-pixel position surrounded by refreshed pixels, mark $p$ as refreshed in $M_{f}$ and stop. Otherwise, set $k=0$ and go to 2).

2. Increase $k$ by 1 . If $k$ is great than $L$, i.e. all the reference frames have been checked, stop. Otherwise, go to 3).

3. If $k$ equals $k_{0}$, go to 2). Otherwise, estimate the MV of $p$ to the $k$ th reference frame based on the constant velocity model, i.e. $M V_{k}=M V_{0} \times k / k_{0}$. Then use $M V_{k}$ to find the corresponding pixel $q_{k}$ in the $k$ th reference frame. If $q_{k}$ lies at an integer-pixel position marked as refreshed, or if $q_{k}$ lies at a subpixel position surrounded by refreshed pixels, replace $p$ by the pixel value of $q_{k}$ and set the status of $p$ as filled_mc in $M_{s}$; stop. Otherwise, go to 2).

\subsection{EC by DC}

Divide the video frame into blocks with size $D \times D, D \in\{4,8,16\}$. Suppose pixel $p$ lies in block $B_{c}$. We first check the eight neighbor blocks of $B_{c}$. If one neighbor lies in an INTRA-MB, $p$ will be refined by the DC of this block, i.e. $D C_{n b}$. In other words, the value of $p$ is changed to

$$
p=w_{d c} \times D C_{n b}+\left(1-w_{d c}\right) \times p,
$$

where $w_{d c}$ is the weighting factor to control the emphasis on DC.

\subsection{EC by SI}

For an INTER-pixel $p$, two nearest refreshed pixels are searched within a window, which is centered at $p$ with size $(2 S+1) \times(2 S+1)$. If there is no or just one such pixel, the condition of $E C$ by $S I$ is not satisfied. Otherwise, suppose the two pixels found are $p_{1}$ and $p_{2}$, with distance $d_{1}$ and $d_{2}$ from $p$, respectively. Then an interpolated value for $p$ from its spatial neighbors can be

$$
\hat{p}=\frac{p_{1} \times d_{2}+p_{2} \times d_{1}}{d_{1}+d_{2}} .
$$

Using weight $w_{s i}$ to control the strength of spatial interpolation, we obtain the final value of $p$ :

$$
p=w_{s i} \times \hat{p}+\left(1-w_{s i}\right) \times p .
$$




\subsection{Summary for the EC algorithm}

If an INTRA-frame $\left(I_{0}\right)$ is lost, all the pixels inside are filled by grey color, i.e. 128 for all the YUV components. Each of the subsequent $N$ frames is decoded and then error concealed as follows until an INTER-frame is lost. Here $N$ is an integer to control the number of frames for EC.

- For the first INTER-frame $\left(P_{1}\right)$, compute the DC of the INTRA-MBs within this frame, i.e. $D C_{\text {intra }}$. Fill the reference frame of $P_{1}$ (the buffer for $I_{0}$ ) and all the INTER-pixels of $P_{1}$ by $D C_{\text {intra }}$. Then for each INTRA-MB in $P_{1}$, use its DC to fill the eight neighboring INTER-MBs.

- For the subsequent frames, the INTER-pixels are error concealed as in Figure 1.

If an INTER-frame is lost, it is reconstructed by copying the previous frame (copy-previous).

\section{Simulation Results}

We use the JVT reference software version 11.0 (baseline profile) for the simulations [13]. The first 300 frames of video sequences Foreman and News (QCIF) are encoded at 7.5fps, and only the first frame is I frame. Two reference frames are used for INTER-prediction. Parameter UseConstrainedIntraPred is set to be 1 in the reference software, i.e. INTER pixels are not used for the prediction of INTRA-MB. And the INTRA-rate for RIR is $3 \%$. The compressed video is supposed to be transmitted though a packet loss channel, and one packet contains the information of one frame. So the loss of one packet will lead to the loss of one entire frame. The simulated packet loss patterns are obtained from [14], with loss rate $P=3 \%, 5 \%, 10 \%$, or $20 \%$. Decoder PSNR is used as the objective measurement, which is computed using the the original uncompressed video as reference. Given a packet loss rate $P$, the video sequence is transmitted 40 times, and the average PSNR for the 40 transmissions is calculated at the decoder side. Three EC algorithms are evaluated, which will act as follows in the case of INTRA-frame loss:

- EC_F0_128: The lost INTRA-frame is filled by grey color, i.e. 128 for all the YUV components. And the subsequent frames are decoded directly.

- EC_F01_DC: The EC approach is the same as $E C_{-} M V_{-} D C_{-} S I$. However, only the lost INTRA-frame and the first INTER-frame are error concealed.

- EC_MV_DC_SI: The proposed algorithm in section 2.4, with parameter $w_{d c}=$ $1 / 2, w_{s i}=1 / 3$ and $S=16$. Suppose the video frames are $I_{0}, P_{1}, P_{2}, P_{3}$, $P_{4} \ldots$. We use $D=16$ for $P_{1}, P_{2}$ and $D=4$ for $P_{i}, i \geq 3 .{ }^{1}$

The lost INTER-frame is error concealed by copy-previous for all these three algorithms.

\footnotetext{
${ }^{1}$ Note that when $E C$ by $S I$ is applied in $E C_{-} M V_{-} D C_{-} S I$, with $w_{d c}=1 / 2$ in Eqn. (1) and $w_{s i}=1 / 3$ in Eqn. (3), an INTER-pixel $p$ is reconstructed by the average of the $D C_{n b}$, the interpolated pixel and the decoded one.
} 

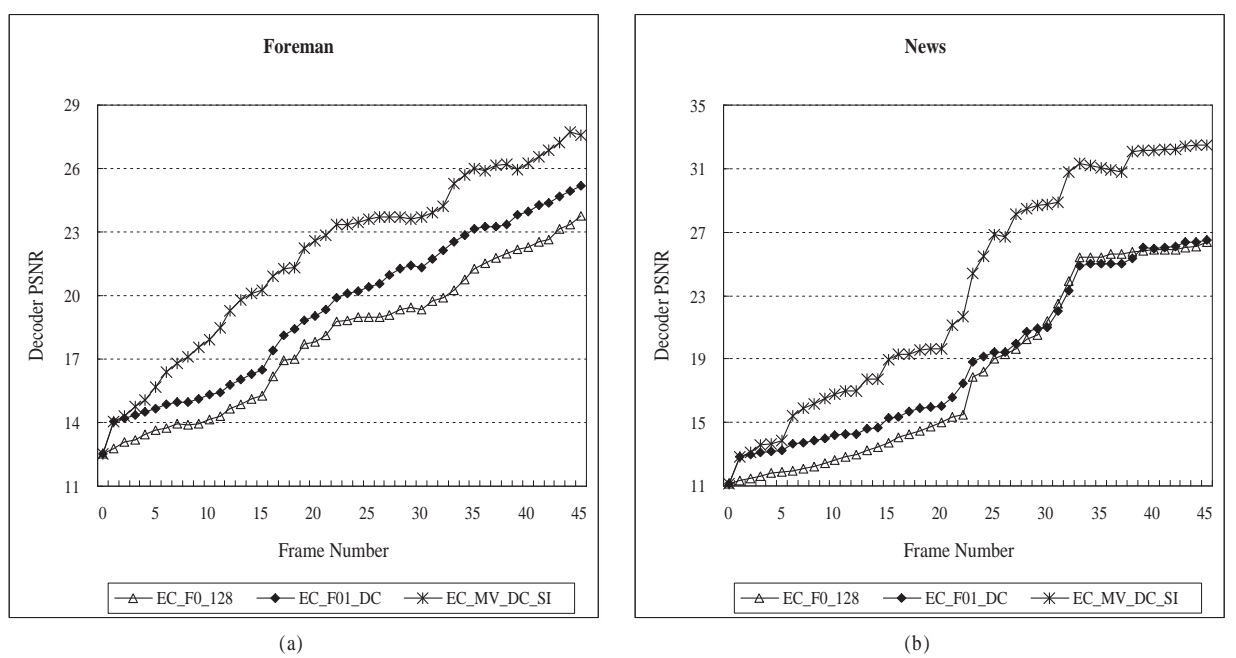

Fig. 2. The decoder PSNR of different EC algorithms for INTRA-frame loss.

Encoder Reconstructed

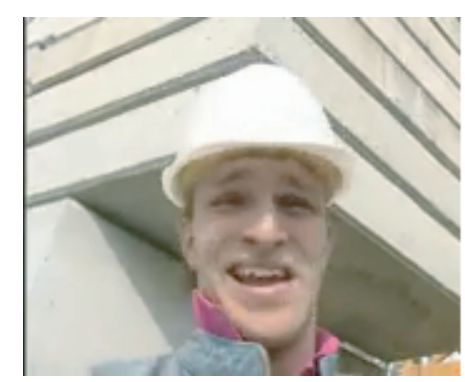

$$
\text { EC_F01_DC }
$$

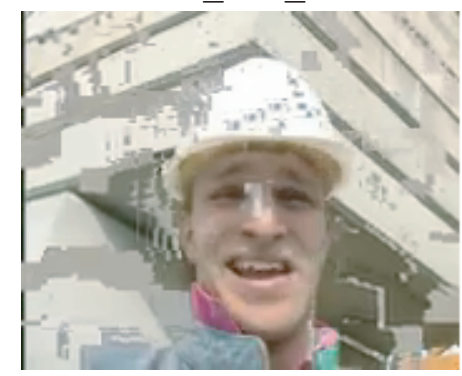

EC_F0_128

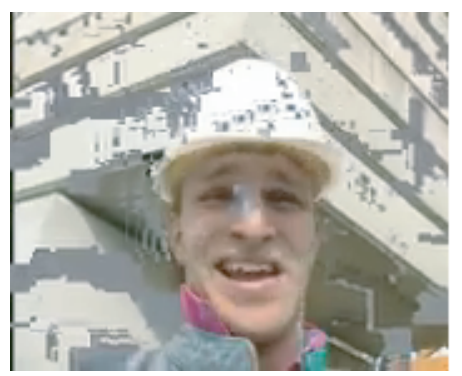

EC_MV_DC_SI

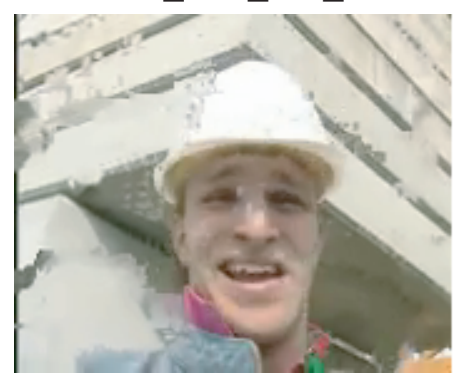

Fig. 3. The 30th INTER-frame of Foreman in different EC algorithms for INTRAframe loss. 
Table 1. The average decoder PSNRs for different loss rate $P$.

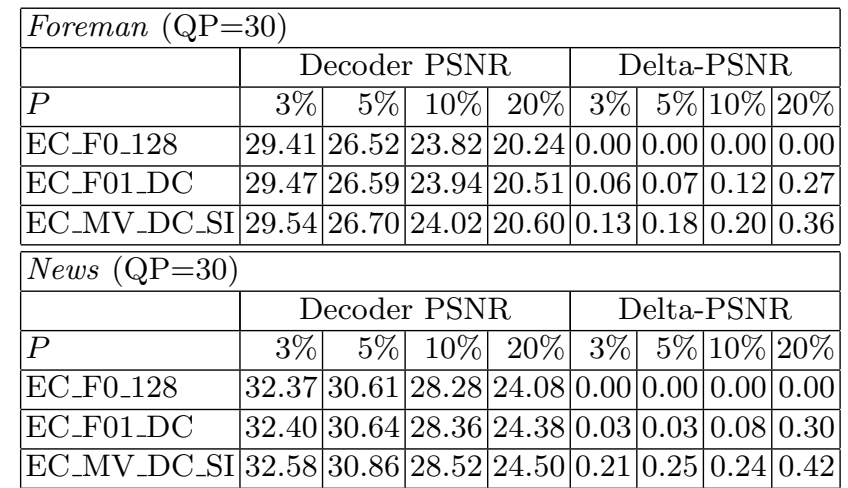

We first simulate the case of INTRA-frame loss, and all the subsequent frames are assumed to be received. Parameter $N$ of $E C_{-} M V_{-} D C_{-} S I$, i.e. the number of frames for EC, is selected to be $N=75$ for a better illustration. Constant QP $(\mathrm{QP}=30)$ is used to encode both Foreman and News. The decoder PSNR is plotted in Figure 2. As shown in the figure, the video quality can be improved by just error concealing the first two frames, i.e. filling with the $\mathrm{DC}$ of received INTRA-MBs. However, with the proposed $E C_{-} M V_{-} D C_{-} S I$ algorithm, much more improvement can be obtained. The reconstructed 30th INTER-frames of Foreman by different algorithms are shown in Figure 3. We can see from the figure that $E C_{-} M V_{-} D C_{-} S I$ can suppress the propagated error more efficiently than the other two algorithms.

The performances of the $\mathrm{EC}$ algorithms under random packet loss conditions are given in Table 1 and Figure 4, with parameter $N=30$ for $E C_{-} M V_{-} D C_{-} S I$. Both INTRA-frames and INTER-frames can be lost according to the packet loss rate $(P)$. Table 1 shows the average decoder PSNRs for different $P$. To give a clearer illustration, we also present the difference between $E C_{-} F 01 \_D C / E C_{-} M V_{-} D C_{-} S I$ and $E C_{-} F 0_{-} 128$ for the same loss rate, as shown in the column named DeltaPSNR. From the table we can see that both $E C_{-} F 01_{-} D C$ and $E C_{-} M V_{-} D C_{-} S I$ can obtain a higher PSNR than $E C_{-} F 0_{-} 128$, and the difference increases with increasing $P$. Figure 4 compares the RD curves of the three $\mathrm{EC}$ algorithms for a given packet loss rate $P$. From the figure we can see that by using $E C_{-} M V_{-} D C_{-} S I$ at $P=5 \%$, we can gain about $0.19 \mathrm{~dB}$ for Foreman and about $0.26 \mathrm{~dB}$ for News, compared to using $E C_{-} F 0_{-} 128$. For $P=20 \%$, about $0.4 \mathrm{~dB}$ can be obtained for both Foreman and News. In addition, for a small loss rate $P$, i.e. $P=3 \%$ or $5 \%$, the performance (decoder PSNR) of EC_F01_DC is closer to $E C_{-} F 0_{-} 128$ than to $E C_{-} M V_{-} D C_{-} S I$. And when $P$ increases, the performance of $E C_{-} F 01 \_D C$ gets closer to $E C_{-} M V_{-} D C_{-} S I$. This can be observed from both Table 1 and Figure 4.

Not that in Table 1 and Figure 4, the gap between EC_MV_DC_SI and $E C_{-} \mathrm{FO}_{-} 128$ is smaller than that in Figure 2. As in the case of INTER-frame 

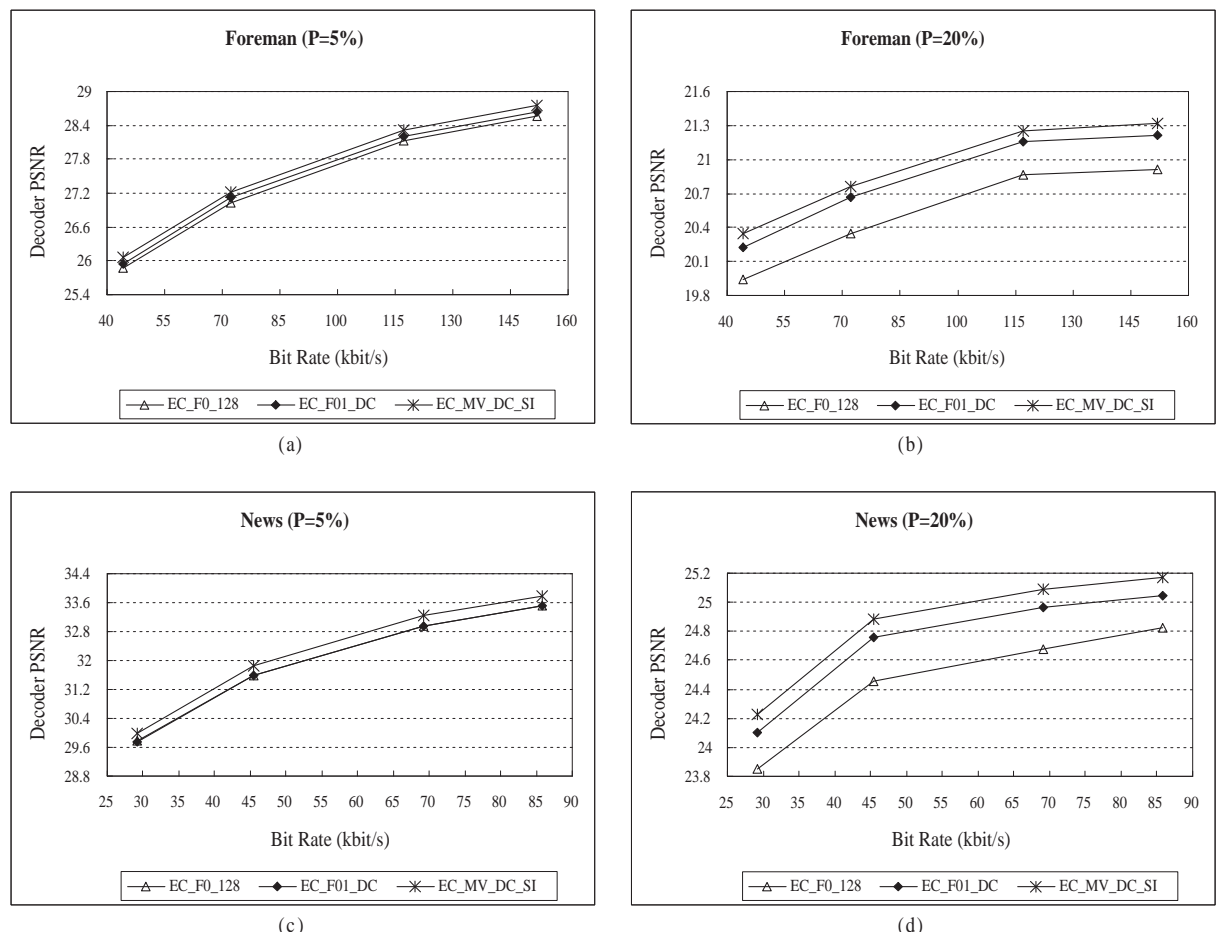

Fig. 4. The RD curves of different EC algorithms with loss rate $P=5 \%$ and $P=20 \%$.

losses, the two algorithms have the same action, i.e. copy-previous, and the advantage of $E C_{-} M V_{-} D C_{-} S I$ over $E C_{-} F 0_{-} 128$ is not obvious. Actually in such conditions, the received INTRA-MBs can also be used to refine the subsequent INTER-frames. We will take this as a future work.

\section{Conclusion}

In this paper, we propose an EC algorithm for INTRA-frame losses over packet loss channels. Both motion compensation and spatial interpolation are used to refine the INTER-pixels in the subsequent frames. As a result, the propagated error can decrease much faster than just error-concealing the lost INTRA-frame.

Acknowledgments. This work has been supported by the Innovation and Technology Commission of the Hong Kong Special Administrative Region, China (project no. GHP/033/05). 


\section{References}

1. Y. Wang and Q. F. Zhu, "Error control and concealment for video communication: a review," in Proc. IEEE, May 1998, pp. 974-997.

2. M. Al-Mualla, C. Canagarajah, and D. Bull, "Multiple-reference temporal error concealment," in Proc. IEEE ISCAS, May 2001, pp. 149-152.

3. Y. Chen, K. Yu, J. Li, and S. Li, "An error concealment algorithm for entire frame loss in video transmission," in Proc. PCS, Dec. 2004.

4. W. Zhu, Y. Wang, and Q.-F. Zhu, "Second-order derivative-based smoothness measure for error concealment in DCT-based codecs," IEEE Trans. Circuits Syst. Video Technol., vol. 8, pp. $713-718$, Oct. 1998.

5. S.-C. Hsia, S.-C. Cheng, and S.-W. Chou, "Efficient adaptive error concealment technique for video decoding system," IEEE Trans. Multimedia, vol. 7, pp. 860 868 , Oct. 2005.

6. W. M. Lam, A. R. Reibman, and B. Liu, "Recovery of lost or erroneously received motion vectors," in Proc. IEEE ICASSP, Mar. 1993, pp. 417-420.

7. C.-Y. Su, S.-H. Tsay, and C.-H. Huang, "Error concealment using directionoriented candidate set and predicted boundary matching criteria," in Proc. IEEE ICIP, Oct. 2006, pp. 2221-2224.

8. S. Belfiore, M. Grangetto, E. Magli, and G. Olmo, "Concealment of whole-frame losses for wireless low bit-rate video based on multiframe optical flow estimation," IEEE Trans. Multimedia, vol. 7, pp. 316-329, Apr. 2005.

9. Z. Wu and J. M. Boyce, "An error concealment scheme for entire frame losses based on H.264/AVC," in Proc. IEEE ISCAS, May 2006, pp. 4463-4466.

10. P. Nasiopoulos, L. Coria-Mendozal, H. Mansour, and A. Golikeri, "An improved error concealment algorithm for intra-frames in H.264/AVC," in Proc. IEEE ISCAS, May 2005, pp. 320-323.

11. H. Wang and J. Lv, "A novel error concealment scheme for intra frames of H.264 video," in Proc. IEEE Int. Workshop VLSI Design 6 Video Tech., May 2005, pp. 300-303.

12. S. Kumar, L. Xu, M. K. Mandal, and S. Panchanathan, "Error resiliency schemes in H.264/AVC standard," Elsevier J. Vis. Commun. Image Represent., vol. 17(2), pp. 425-450, Apr. 2006.

13. Jvt reference software, version 11.0. [Online]. Available: http://iphome.hhi.de/ suehring/tml/download/

14. S. Wenger, "Error patterns for internet experiments," in ITU-T SG16 Doc. Q15I-16r1, Oct. 1999. 